\title{
Fostering Collaboration in Kindergarten through an Augmented Reality Game
}

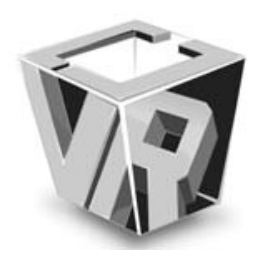

\author{
Pedro Campos ${ }^{1}$, Sofia Pessanha ${ }^{2}$ and Joaquim Jorge ${ }^{3}$ \\ ${ }^{1}$ University of Madeira, Madeira Interactive Technologies Institute and INESC-ID Lisbon \\ ${ }^{2}$ University of Madeira, Portugal \\ ${ }^{3}$ INESC-ID Lisbon, Portugal
}

\begin{abstract}
Kindergarten children are a very special class of users, since they are in a primary stage of life, when they must learn how to live in society, e.g. to listen and respect the others' opinions, share the same objects and also help each other. This study presents an Augmented Reality game, in which kindergarten children were able to collaborate in a spontaneous way supported by motivation, enjoyment and curiosity. This game allows children to explore concepts like the animals and the environments they live in by using Augmented Reality markers and a wooden board. These markers are the game pieces and through them children are able to manipulate $3 D$ virtual models.

Experiments were performed with several classes of students in different schools. Results suggest that the game is effective in maintaining high levels of motivation and collaboration among children, particularly when using immediate feedback.
\end{abstract}

Index Terms - Augmented reality; Interactive learning systems; Collaboration;

\section{INTRODUCTION}

Using games as a way for better educating children is becoming increasingly popular because children are moving towards a new level of interaction with technology and there is a need to approach them towards the educational contents. This can be done through the use of novel, more attractive technologies.

The power of digital games as educational tools is, however, well understood. Games can be successfully used for teaching science and engineering better than lectures [1], and e.g. Mayo and colleagues even argued they could be the "cure for a numbing 200-person class.” [1]. Games can also be used to teach a number of very different subjects to children all ages. For instance Gibson describes a game aimed at teaching programming to pre-teens school children [2]. Belotti and colleagues [5] describe an educational game using a state-of-the-art commercial game development approach, and enriched the environment with instances of developed educational modules. The research goals for these approaches are essentially to exploit the potential of computers and reach a demographic that is traditionally averse to learning.

On a more specific line, there is also interesting research on using Augmented Reality (AR) games in the classroom. From high-school mathematics and geometry [3] to interactive solar

Manuscript Received on September 20, 2010.

E-mail: pedro.campos.pt@gmail.com systems targeted at middle school science students [4], the range of applications is relatively broad.

However, there is a clear lack of solutions and studies regarding the application of these technologies with kindergarten children, who are aged 3-5 years old and therefore have different learning objectives.

In this paper, we present a tangible user interface for an augmented reality game specifically targeted at promoting collaborative learning in kindergarten. The game's design involved HCI researchers (the authors), kindergarten teachers and 3D designers. We evaluated the system during several days in tow different local schools and we recorded the children's reactions, behaviors and answers to a survey we also conducted.

Instead of developing a computer program using traditional input techniques (mouse and keyboard), this research presents a novel user interface for learning kindergarten subjects. The motivation is essentially to bring something from the real world and couple that with virtual reality elements, accomplishing the interaction using our own hands, thus, children don't need to have previous experience using computers in order to use this system. The interface is, essentially, a symbiosis of traditional cardboard games with digital technology.

The rationale for our approach is twofold. First, Papert [7] refers that "learning is more effective when the apprentice voluntarily engages in the process". Motivating the learners is therefore a crucial factor to increase the possibility of action and discovery, which in turn increases the capacity of what some researchers call learning to learn. In this sense, the novel constructionist-learning paradigm aims to adapt and prepare tomorrow's schools to the constant challenges faced by a society, which is currently embracing and accelerating pace of profound changes. Augmented reality [6] and tangible user interfaces [8] fit nicely as a support method for this kind of learning paradigm since they promote higher levels of motivation and engagement.

Secondly, kindergarten children's educational goals and needs are much different and much more based around collaborative notions such as respecting their colleagues, interacting with them, solving problems together and other society values. These principles are much more important in kindergarten than e.g. learning a given subject. Designing novel games for kindergarten must therefore promote good levels of effective, fruitful collaboration.

Kindergarten is the first stage of basic education and is the beginning of the lifelong educational process. It should favor 
the children's education and balanced development with the goal of successfully integrating the child in the society as a free, autonomous human being with solidarity. In this stage of life, children have the opportunity to learn attitudes, expressions, languages and comprehension of the world they live in.

It is important to emphasize that kindergarten's educational contents are essentially based on what children already know and what they have already learned, and therefore the focus is on creating conditions for successful future learning experiences [15]. It is up to the kindergarten teacher to organize the educational process in such a way that it fits to each child's characteristics, stimulating each child's evolution in the whole group. This is the reason why we chose to focus our study on design issues that could influence collaborative behaviors, instead of simply studying the effects of the game on motivation and learning levels.

The remainder of this paper is organized as follows: the next section describes related work with a particular emphasis on research approaches using augmented reality technology and approaches that promote collaboration in the classroom. The next Section describes the developed game. Section "Evaluation” describes the procedure and participants in the experimental evaluation, which was divided into learning, motivation and collaboration issues. We proceed to a discussion around implications of this study and finally Section "Conclusions and Future Work” outlines new avenues of research for this field.

\section{RELATED WORK}

Technology today provides exciting new possibilities to approach children to digital contents. There are numerous areas where Augmented Reality (AR) can be applied, ranging from more serious areas to entertainment and fun. Thus, the process of viewing and manipulating virtual objects in a real environment can be found in many applications, especially in the area of education and training which are very promising applicants, since it is often necessary to use resources enabling a better view of the object under study. Other applications include the creation of collaborative environments in AR, which consist of multi-user systems with simultaneous access where each user views and interacts with real and virtual elements, each of their point of view.

Given the scope of our work, we divide the review of the literature into two broad aspects: the use of augmented reality technology in the classroom, and approaches targeted at promoting collaboration in the classroom by means of novel technology - not necessarily based in augmented reality.

\subsection{Augmented Reality in the Classroom}

The use of augmented reality systems in educational settings, per se, is not novel. Shelton and Hedley [6] describe a research project in which they used augmented reality to help teach undergraduate geography students about earth-sun relationships. They examined over thirty students who participated in an augmented reality exercise containing models designed to teach concepts of rotation/revolution, solstice/equinox, and seasonal variation of light and temperature, and found a sig- nificant overall improvement in student understanding after the augmented reality exercise, as well as a reduction in student misunderstandings.

Some other important conclusions about this system were that AR interfaces do not merely change the delivery mechanism of instructional content: They may fundamentally change the way that content is understood, through a unique combination of visual and sensory information that results in a powerful cognitive and learning experience [6].

Simulations in virtual environments are becoming an important research tool for educators [9]. Augmented reality, in particular, has been used to teach physical models in chemistry education [10]. Schrier evaluated the perceptions regarding these two representations in learning about amino acids. The results showed that some students enjoyed manipulating AR models by rotating the markers to observe different orientations of the virtual objects [10].

Construct3D [9] is a three-dimensional geometric construction tool specifically designed for mathematics and geometry education. In order to support various teacher-student interaction scenarios, flexible methods were implemented for context and user dependent rendering of parts of the construction. Together with hybrid hardware setups they allowed the use of Construct3D in classrooms and provided a test bed for future evaluations. Construct3D is easy to learn, encourages experimentation with geometric constructions, and improves spatial skills [9].

The wide range of AR educational applications also extend to physics. Duarte et al. [11] use AR to dynamically present information associated to the change of scenery being used in the real world. In this case, the authors perform an experiment in the field of physics to display information that varies in time, such as velocity and acceleration, which can be estimated and displayed in real time.

The visualization of real and estimated data during the experiment, along with the use of AR techniques, proved to be quite efficient, since the experiments could be more detailed and interesting, thus promoting the cognitive mechanisms of learning.

Min-Chai Hsieh and Jiann-Shu Lee [19] developed a system named ARELS (Augmented Reality English Learning System) to help kindergarten's children learn English. They made a card for each letter of the alphabet and each of them shows a 3D image of a word started with the letter in question and can also display text, images, music, videos and animations. They also proposed a method reducing complexity and increase capacity of designing AR markers based on permutation and combination's concept.

Campos and Freitas [20] developed a system named SMART (System of Multimodal Augmented Reality for Teaching) targeted at children in elementary school. It was a game based on the categories of animals and transports. The conclusions of this study was that AR can help to increase motivation and thus student learning, contributing positively for the learning of students with more learning difficulties [20].

The use of AR in formal education could prove a key component in the learning environments of the future. These environments will be abundantly populated with a blend of hardware and software applications. However, relatively little is known about the potential of this technology to support teach- 
ing and learning with groups of young children in the classroom, in particular in kindergarten. Except for Kerawalla et al. [12] and some other studies, few researchers actually present results of experiments using this kind of technology in real life schools. Kerawalla et al. [12] analyzed teacher-child dialogues in a comparative study between use of an AR virtual mirror interface and more traditional science teaching methods for 10 -year-old children. This study revealed that the children using AR were less engaged than those using traditional resources. This shows that using AR alone is not only insufficient to keep children engaged, it could even reduce their level of engagement, which proves the challenge posed to researchers when trying to deploy and study systems like the one we propose.

\subsection{Promoting Collaboration}

Promoting collaborating behaviors is crucial in the kindergarten educational context. Therefore, we briefly analyze approaches that use technology as a way to achieve higher levels of collaboration in the classroom.

Children communicate and learn through play and exploration [16]. Through social interaction and imitating one another, children acquire new skills and learn to collaborate with others. This is also true when children work with computers.

Using traditional mouse-based computers, and even taking into consideration that two or more children may collaborate verbally, only one child at a time has control of the computer. The recognition that group work around a single display is desirable has led to the development of software and hardware that is designed specifically to support this. The effect of giving each user an input device, even if only one could be active at a time was then examined and significant learning improvements were found [17].

Stewart et al. [18] observed that children with access to multiple input devices seemed to enjoy an enhanced experience, with the researchers observing increased incidences of student-student interaction and student-teacher interaction as well as changing the character of the collaborative interaction. The children also seemed to enjoy their experience more, compared with earlier observations of them using similar software on standard systems.

There are also studies about the design of user interfaces for collaboration between children [14]. Some results present systems which effectively supported collaboration and interactivity that children enjoyed, and were engaged in the play [14].

Kannetis and Potamianos [13] investigated the way fantasy, curiosity, and challenge contributes to the user experience in multimodal dialogue computer games for preschool children, which is particularly relevant for our research. They found out that fantasy and curiosity are correlated with children's entertainment, while the level of difficulty seems to depend on each child's individual preferences and capabilities [13]. One issue we took into account when designing our AR game for kindergarten was that preschoolers become more engaged when multimodal interfaces are speech enabled and contain curiosity elements. We specifically introduced this element in our design, and confirmed the results described in [13].

\section{THE GAME}

As with any game, the solution space dimension was very high, so we collaboratively designed the game with kindergarten teachers, focusing on a biodiversity theme, using traditional book-based activities as a starting point.

The developed system was based on a wooden board containing nine divisions where children can freely place the game's pieces. The pieces are essentially based on augmented reality markers. Several (experienced) kindergarten teachers provided us with a learning objective and actively participated in the entire game's design. For instance, they listed a series of requirements that any game or educational tool should comply when dealing with kindergarten children. They can be aged from 3 to 5 years old, and therefore have different teaching and caring needs, when compared with older children or other types of users. Among the most important requirements were:

- Promote respectful collaborative behaviors like giving turns to friends, pointing out mistakes and offering corrections;

- Promote learning of the given subject.

- Promote a constructivist approach, where children learn by doing and by constructing solutions;

- The previous requirement also implied that the physical material of the tangible interface had to be resistant and adequate to manipulation by the group of children;

In our case, the learning objective was the study of animals and the environments (sea, rivers, land and air) they live in. Each division of the board's game contains a printed image of a given environment.

Given the manipulative nature of such game, the game's pieces had to be made from a special material, which is particularly suited for children, a flexible but robust material. Each of the game's pieces displays a 3D animal that can be manipulated, as in a regular augmented reality setting. The board also contains a fixed camera, which processes the real time video information. Fig. 1 illustrates the overall setting of the system, which can be connected to any kind of computer and display. In the figure, we show the system connected to a laptop, but during classroom evaluation we used a projector, to facilitate collaborative learning.

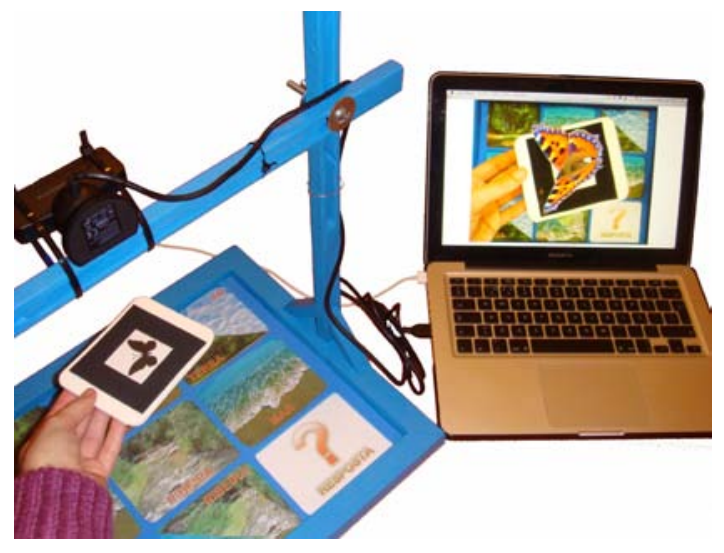

Fig. 1. The developed system, when used in a LCD display configuration.( Color Plate 7)

The goal of the game is to place all the markers (game board pieces representing animals) in the correct slot of the board. We 
only give feedback about the correctness of the placement of pieces in the end, when the player places a special marker that is used for that purpose, i.e. a "show me the results" marker. Two different versions of the game were developed, to assess the impact of the feedback's immediacy on the children's levels of collaboration: a version where feedback can be freely given at any time (whenever children place the special marker to see the results, as shown in Fig. 2); and a version where feedback is only given at the end of the game, i.e. when all the pieces have been placed in the board (again, by placing the special marker).

Fig. 2 shows a screenshot of what children see displayed in the screen. The markers display 3D animals, which can be freely manipulated. The animals that are correctly placed have a green outline, incorrectly placed animals show a red outline. Following the teachers' suggestions, we also added audio feedback, with pre-recorded sentences like "That's not right, try it again!” This encouraged children, especially when positive reinforcement was given in the form of an applause sound.

The game also features a detailed logging mechanism with all actions recorded with timestamps. This was developed as an aid to evaluating the effects on collaboration levels. The system logs the completion times of each game, the number of incorrectly placed markers, the number of feedback requests (which can be considered the number of attempts to reach a solution), and other variables.

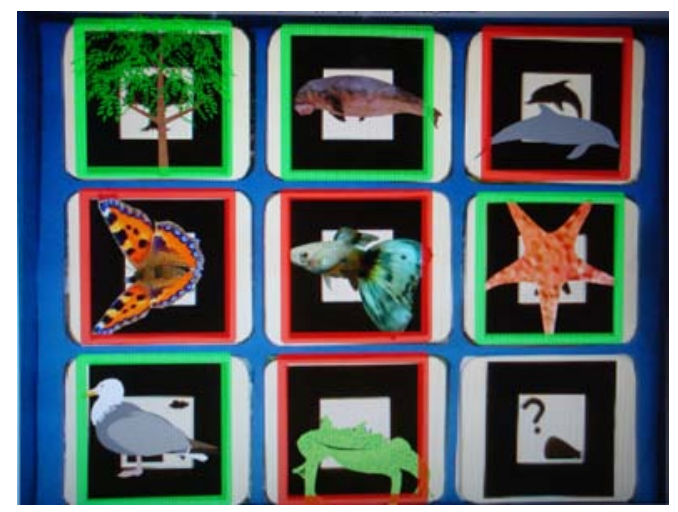

Fig. 2. The game's screen, showing feedback as a red or green border around the animals.( Color Plate 8)

\section{EVALUATION}

Researchers are advised that the impact of a technology cannot be fully understood without considering the whole educational context [21]. Therefore, we undertook a mixed-method approach in this study. A combination of quantitative (pre and post tests) and qualitative (observations, interviews, open-ended questions, and digital logs of the actions) methods permitted a grounded understanding of the impact of the game in (i) learning, (ii) motivation and (iii) collaboration levels. Although we present the method and results for all three items (learning effectiveness, motivation levels and collaboration levels) our real scope was about collaboration levels, i.e. we wanted to investigate how a game like this can promote higher levels of collaborative behaviors.

\subsection{Method}

Two different experiments were performed using the AR game as a prototype for answering two research hypotheses: the role of immediate feedback and size of the display on the levels of collaboration; and the effects on learning and motivation levels. Mixing video capture of both the computer screen and the children facilitated the analysis, as well as including a logging mechanism in the software.

The first step was to make sure the prototypes were well tested. For this, we used the University's labs, under different lighting conditions - augmented reality won't work in totally dark environments because it is based on the camera's recognition of the black and white printed markers. It also fares poorly when specific lighting conditions cause reflections on the markers. We also informally tested the prototype with some users.

The main procedure was divided into two issues and two different experiments aimed at studying those two issues. Those issues were (i) the effects the game had on learning, and (ii) the effects different design variables had on collaboration levels. We also investigated the effect on children's motivation, which were high as we expected, since any game or new technology has the potential to motivate the children. Since a motivation increase was already expected, and also since the effect on learning is difficult to measure in kindergarten, we chose to focus our study on collaboration levels. This was also due to the importance that promoting successful collaborations has on the kindergarten environment. Teachers even told us "it was more advantageous if such a game could promote respectful and fruitful collaborations among children than being able to positively influence their learning”.

We wanted, however, to ensure the game would do no harm to children's learning, i.e. it was desirable if the game could also serve as an effective teaching aid. Therefore, we started out by performing an experiment in an initial school aimed at knowing if the game really aids children in novel knowledge acquisition.

Each class went through three different phases: a pretest phase, where students answered a random set of questions about the subject being taught (the environments where animals live), without being taught anything about it. Then came the learning phase itself. It consisted of playing the game in groups of four children. The next day, a posttest was performed, and we measured the difference in results, which will be described in the next section.

A second experiment (in a different school and with different participants) was performed to assess the collaboration levels, and to investigate the influence of the following variables on those levels of collaboration: (i) the display size (using a projector versus using a 16-inch LCD screen) and (ii) the immediacy of the feedback (providing feedback only after all pieces are placed versus providing feedback at anytime).

During both experiments, and whenever the game play started, a screen recording software was automatically launched and kept running in background. Therefore, the children (without knowing, but with consent from parents and teachers) were being digitally videotaped while they played the game. The first author later analyzed thoroughly all the recorded 
movies for gathering reliable results on motivation and collaboration levels as well as the overall class reaction and use of the system, which are described in the next section.

The participants (and the school) of the experiment aimed at assessing the effects on learning were different from the participants and school of the second experiment, aimed at investigating effects on collaboration levels.

The participants of the first experiment were twenty-two children aged from 5 to 6 years old (9 female) who interacted with the system in a collaborative setting using a projector as display device, as shown in Fig. 3. Since teachers informed us about their limited time of attention focus, which is about 45 to 60 minutes, we split them into groups of 4 for each gaming session.

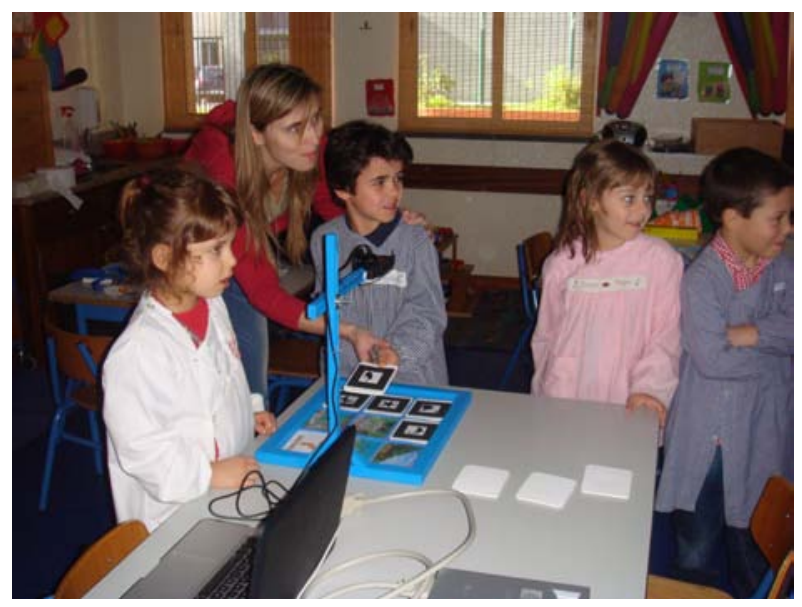

Fig. 3. Children interacting with the system.( Color Plate 9)

The participants of the second experiment were thirty-six different children aged from 3-5 years old (18 female), who played the game in groups of four or five. There were four groups of four and there were four groups of five.

Both groups were balanced in terms of computer skills and gender. They had never contacted with the system before and went through a brief (3-5 minutes) introductory session with the teachers.

\subsection{Results}

\subsection{1) Motivation}

Motivation was essentially measured by observation, video recordings' analysis and questionnaires handed out to teachers so that they could question children's levels of motivation after the sessions.

As a baseline, we studied children's interactions with a smartboard that featured a similar game (in a powerpoint-like version), and we observed that the most active children could not concentrate themselves. Concretely, while one of the children was interacting on the board, the others were seated on the floor, and we observed that they had much difficulty in staying concentrated and quiet. This wasn't the case with the AR game.

Motivation was also evident during playground times, when most children chose not to go outside and play, but rather kept on playing with the AR game.

Finally, motivation levels were high because children never gave up the game. Even when feedback showed them they were very wrong, nobody quit the game until reaching the solution. In fact, there is an incentive to discovery, since the game appeals to children's curiosity by allowing them to view each marker once at a time.

Through video analysis, we observed that non-attentive behavior (such as looking away from the computer screen) was very low in all testing conditions, when playing the game in groups of four or five.

\subsection{2) Collaboration}

The children's overall reaction to the system was very positive, in both experiments, and most importantly, we concluded that the system didn't make the learning process go wrong. However, since we were interested in studying collaboration levels, we evaluated the game in a different school with different children, under a collaboration perspective.

We measured collaboration levels under two different conditions, in a between-subjects experimental design: using a projector versus using an LCD as a display, and using immediate feedback versus providing feedback only at the end of the markers placement in the game board.

Feedback Immediacy. Our research question \#1 was: Can the possibility of providing immediate feedback increase the levels of collaboration among children playing this game?

To test this hypothesis, we recorded the game's completion times, and manually analyzed the video recordings of the experiment in order to measure the dependent variable (collaboration) in the following quantitative manner: (i) number of collaborative comments made by children, (ii) number of constructive collaborative corrections made by children, including pointing gestures, and (iii) number of attempts made until reaching a solution. There were seven groups of children in this experiment, totaling $\mathrm{N}=7$ for the statistic measurements.

Completion times (in minutes) in the immediate feedback version of the game were higher $(M=5.79, S D=1.07)$ than in the version where feedback is only available after placement of the markers $(M=4.43, S D=0.85)$ and this difference was considered statistically significant $(\mathrm{t}(12)=2.63$, $\mathrm{p}=0.021)$. Although completion time is not a measure of collaboration, the subsequent results from the collaborative interactions suggest that the higher completion time for the immediate feedback version was caused by a higher number of collaborative interactions.

Regarding the number of collaborative comments made, i.e. comments children made specifically regarding the game playing process, we observed these were also higher in the immediate feedback version $(\mathrm{M}=8.86, \mathrm{SD}=5.08)$ than the other version ( $\mathrm{M}=1.85, \mathrm{SD}=1.07)$, and this difference was considered to be very statistically significant. $(\mathrm{t}(12)=3.57, \mathrm{p}=0.004)$. Children made significantly more collaborative comments by playing with the immediate feedback version.

Regarding the number of corrective actions (e.g. switching a already placed marker from one placement to another) and pointing gestures, we again observed that the number was higher in the immediate feedback version $(\mathrm{M}=8.0, \mathrm{SD}=4.32)$ than in the other version $(\mathrm{M}=2.0, \mathrm{SD}=1.41)$. Again, this difference was very statistically significant $(t(12)=3.49, p=0.005)$.

Finally, the number of attempts made until reaching a solution was also higher in the immediate feedback version $(\mathrm{M}=2.0$, $\mathrm{SD}=1.41)$ than the other $(\mathrm{M}=2.0, \mathrm{SD}=1.41)$, with $\mathrm{t}(12)=18.9$, 
$\mathrm{p}=0.0001$.

These results suggest that immediate feedback may play a role in increasing the number of collaborative behaviors and interactions among kindergarten children.

Also, according to teachers' feedback which was drawn from semi-structured interviews conducted after the evaluations, the children effectively collaborated, since they helped each other by changing the position of the game's physical pieces, making comments, and playing in an orderly manner. The game's board acted like a communication platform between children of each group.

Display Size. Our research question \#2 was: Can the size of the display influence the levels of collaboration among children playing this game?

To test this hypothesis, we proceeded the same way, but varying the size of the display. We used a common projector, as illustrated in Figure 4, with four groups of children, and we used a 16-inch LCD with four other groups. These were all different children than the ones of the previous experiment.

Contrarily to our expectations, completion times were slightly higher $(\mathrm{M}=5.61, \mathrm{SD}=1.82)$ in the small display (LCD) version than in the large display (projector) version ( $M=5.33$, $\mathrm{SD}=0.88$ ). However, the difference was not significant $(\mathrm{t}(6)=0.27, \mathrm{p}=0.791)$.

The difference in the number of comments made by children using the small display $(\mathrm{M}=7.25, \mathrm{SD}=7.27)$ versus the large display $(\mathrm{M}=8.25$, $\mathrm{SD}=4.57)$ was also not significant $(\mathrm{t}(6)=0.24$, $\mathrm{p}=0.82$ ).

The number of corrective actions and gestures was higher in the large display version $(\mathrm{M}=8.5, \mathrm{SD}=5.06)$ than in the small display ( $M=5.75, S D=4.57)$. However the difference was not considered statistically significant $(\mathrm{t}(6)=0.81, \mathrm{p}=0.451)$.

\section{DISCUSSION AND IMPLICATIONS}

The results obtained so far indicate that using our augmented reality system is a positive step forward towards achieving the goal of reducing the distance between children and knowledge, by learning through play.

The system has a very positive impact on the whole class collaboration. This is much harder than it seems, since kindergarten children have very low attention cycles. They get distracted very often, and they have trouble collaborating in an orderly manner. An important contribution from this paper, in terms of design issues that promote collaboration, is the importance of providing immediate feedback in virtual reality games such as the one we have developed. It is crucial that designers targeting kindergarten children are capable of exploiting the innate curiosity in these tiny users in order to achieve good levels of collaborative interactions.

Motivation, enjoyment and curiosity are important ingredients for any kind of educational game, but they are even more important when it comes to kindergarten user interfaces. Interaction with tangible board pieces (the AR markers) may be well suited to very young children because of their physicality, but this is could not be sufficient to achieve good levels of motivation and collaboration.

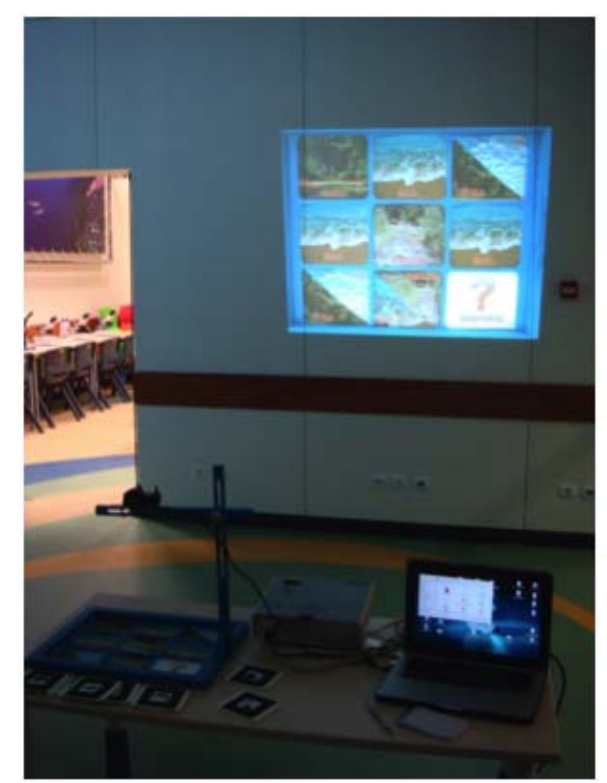

Fig. 4. Configuration of the system with the projector and the LCD (only one of each was used at a given time).

\section{CONCLUSION}

Augmented reality technology and tangible interfaces are well accepted by today's kindergarten children and by their teachers as well. Large projection screens and a good blend of the physical game pieces with their virtual ones can prove effective for increasing motivation and collaboration levels among children. In the learning field, we also concluded that by playing the game the children's number of wrong answers decreased, which suggests the game could help kindergarten children to learn simple concepts.

Since kindergarten children loose the focus of their attention frequently, specially with a game, we feared that the game could harm the learning process. These results suggest that the game didn't make any harm to that process, since the next day's posttest results showed a positive improvement. According to teachers' feedback, the game looks like a promising way to complement the traditional teaching methods.

About motivation, we observed high levels of motivation while children played the game because most of them were clearly motivated, e.g. they never gave up the game until they found the solution. Curiosity was another driving factor towards motivation. Children wanted to see all the 3D animals but for that to happen, they had to wait until all markers were placed. In terms of maintaining motivation, this was a crucial design issue.

This research focus was around promoting collaboration. We analyzed several variables such as the number of collaborative comments made by children, number of constructive collaborative corrections made by children, including pointing gestures and the number of attempts made until reaching a solution. Results suggest that immediate feedback played an important role, increasing the number of collaborative behaviors and interactions among kindergarten children.

We also studied the impact of display size, but the results 
showed that differences were not significant, although by observation, and also according to teachers' feedback, the larger display seemed to better promote collaboration levels than the smaller display. Future work should consist of expanding the experiment in order to better assess the role played by the display size in collaboration levels. Future work will also include more tests with different schools, as well as investigating other features and design issues that could positively influence collaboration in kindergarten.

\section{ACKNOWLEDGEMENT}

We thank the students who helped us during the extensive observation period, and to the kindergarten teachers of both schools (omitted for blind-review purposes). This work was supported by FCT (INESC-ID multiannual funding) through the PIDDAC Program funds.

\section{REFERENCES}

[1] M. J. Mayo, (2007). Games for science and engineering education. Communications of the ACM, 50, 7 (Jul. 2007), pp. 30-35.

[2] J. P. Gibson, (2003). A noughts and crosses Java applet to teach programming to primary school children. In Proceedings of the 2nd international Conference on Principles and Practice of Programming in Java, PPPJ, vol. 42. Computer Science Press, New York, NY, pp. 85-88.

[3] H. Kaufmann and D. Schmalstieg, (2002). Mathematics and geometry education with collaborative augmented reality. In ACM SIGGRAPH 2002 Conference Abstracts and Applications, ACM, New York, NY, 37-41.

[4] P. S. Medicherla, G. Chang and P. Morreale, (2010). Visualization for increased understanding and learning using augmented reality. In Proceedings of the international Conference on Multimedia information Retrieval, MIR'10. ACM, New York, NY, pp. 441-444.

[5] F. Bellotti, R. Berta, A. D. Gloria and L. Primavera, (2009). Enhancing the educational value of video games. Computers in Entertainment, 7, 2 (Jun. 2009), pp. 1-18.

[6] B. Shelton and N. Hedley, (2002). Using Augmented Reality for Teaching Earth-Sun Relationships to Undergraduate Geography Students. In The First IEEE International Augmented Reality Toolkit Workshop, Darmstadt, Germany, September 2002, IEEE Catalog Number: 02EX632 ISBN: 0-7803-7680-3.

[7] S. Papert, (1996). The Connected Family: Bridging the Digital Generation Gap. Longstreet Press.

[8] E. Sharlin, B. Watson, Y. Kitamura, F. Kishino and Y. Itoh, (2004). On tangible user interfaces, humans and spatiality. Personal Ubiquitous Computing, 8, 5 (Sep. 2004), 338-346.

[9] S. Tettegah, K. Taylor, E. Whang, S. Meistninkas and R. Chamot, (2006). Can virtual reality simulations be used as a research tool to study empathy, problems solving and perspective taking of educators?: theory, method and application. International Conference on Computer Graphics and Interactive Techniques, ACM SIGGRAPH 2006 Educators program, Article No. 35.

[10] K. Schrier, (2006). Using augmented reality games to teach 21st century skills. In International Conference on Computer Graphics and Interactive Techniques, ACM SIGGRAPH 2006 Educators program.

[11] M. Duarte, A. Cardoso and E. Lamounier Jr, (2005). Using Augmented Reality for Teaching Physics. WRA'2005 - II Workshop on Augmented Reality, pp. 1-4.

[12] L. Kerawalla, R. Luckin, S. Seljeflot and A. Woolard, (2006). "Making it real": exploring the potential of augmented reality for teaching primary school science. Virtual Reality, Volume 10, Numbers 3-4, December 2006 , pp. 163-174.

[13] T. Kannetis and A. Potamianos, (2009). Towards adapting fantasy, curiosity and challenge in multimodal dialogue systems for preschoolers. In
Proceedings of the 2009 international Conference on Multimodal interfaces, ICMI-MLMI'09. ACM, New York, NY, 39-46.

[14] D. Africano, S. Berg, K. Lindbergh, P. Lundholm, F. Nilbrink, and A. Persson, (2004). Designing tangible interfaces for children's collaboration. In CHI '04 Extended Abstracts on Human Factors in Computing Systems, CHI '04. ACM, New York, NY, 853-868.

[15] N. Brosterman, (1997). Inventing Kindergarten. Harry N. Adams Inc.

[16] Sutton-Smith, B. Toys as culture. New York: Gardner Press, 1986.

[17] K. M. Inkpen, K. S. Booth, M. Klawe and J. McGrenere (1997). The Effect of Turn-Taking Protocols on Children's Learning in Mouse- Driven Collaborative Environments. In Proceedings of Graphics Interface (GI 97), Canadian Information Processing Society, pp. 138-145.

[18] J. Stewart, E. M. Raybourn, B. Bederson and A. Druin, (1998). When two hands are better than one: Enhancing collaboration using single display groupware. In Proceedings of Extended Abstracts of Human Factors in Computing Systems (CHI '98).

[19] Hsieh, Min-Chai. and Lee, Jiann-Shu., (2008). AR Marker Capacity Increasing for Kindergarten English Learning. National University of Tainan, Hong Kong.

[20] R. Freitas and P. Campos, (2008). SMART: a System of Augmented Reality for Teaching 2nd grade concepts. In Proceedings of HCI 2008, Culture, Creativity, Interaction.

[21] G. Salomon, (1990). Studying the flute and orchestra: Controlled experimentation vs. Whole classroom research on computers. International Journal of Educational Research, 14, pp. 37-47.

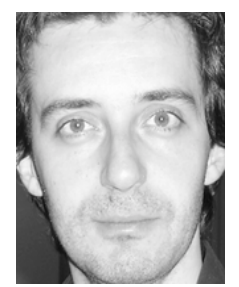

Pedro Campos is an Assistant Professor at the University of Madeira, Portugal, and an Invited Researcher at the Visualization and Intelligent Multimodal Interfaces Group, INESC ID Lisbon. He is also the Program Director for the BSc. in Computer Science and a founding member and Secretary Officer of the IFIP 13.6 Working Group on Human Work Interaction Design. From 2011-onwards he is Vice-President of the Madeira Interactive Technologies Institute. He holds a $\mathrm{PhD}$ in Human-Computer Interaction from the University of Madeira (2006) and a MSc in Intelligent Multimedia Systems from the Technical University of Lisbon (2003).

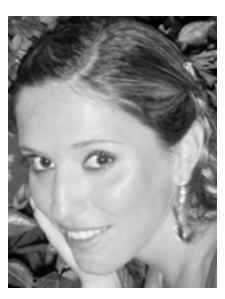

Sofia Pessanha is a MSc thesis student in the University of Madeira, Portugal. She hold a BSc in Computer and Software Engineering also from the University of Madeira. Since 2009 she has been interested in researching about novel interaction paradigms applied to learning in the context of kindergarten schools.

Her other research interests include: user interface design, requirements engineering and software design methodologies.

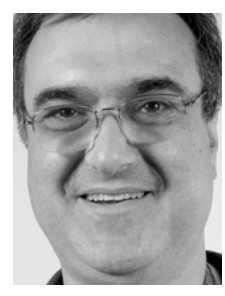

Joaquim A. Jorge is Full Professor at the Computer Science and Engineering Department at IST / TU Lisbon, Portugal, where he teaches Human-Computer Interaction and Computer Graphics. He received PhD and MSc degrees in Computer Science from Rensselaer Polytechnic Institute, Troy, NY, in 1995 and a BsEE from IST/UTL in 1984. He is Editor in Chief of Computers \& Graphics Journal since 2007, is Editorial Board member of six other journals has been chair or co-chair of many international conferences, and has served on the program committees of over 130 international conferences. His interests are in Calligraphic and Multimodal User Interfaces, Visual Languages and Pattern Recognition techniques applied to Human-Computer Interaction. He is author or co-author of over 160 papers published in peer-reviewed international conferences and publications. He was elected Fellow of the Eurographics Association in 2010. 